Published in final edited form as:

Drug Discov Today Dis Models. 2013 ; 10(3): e137-e1743. doi:10.1016/j.ddmod.2013.10.001.

\title{
MicroRNAs and Polycystic Kidney Disease
}

\author{
Lama Noureddine, Sachin Hajarnis, and Vishal Patel \\ Division of Nephrology, Department of Internal Medicine, University of Texas Southwestern \\ Medical Center, Dallas, Texas, USA
}

\begin{abstract}
Polycystic kidney disease (PKD), the most common genetic cause of chronic renal failure, is characterized by the presence of numerous fluid-filled cysts in renal parenchyma. Despite recent progress, no FDA-approved therapy is available to retard cyst growth. Here, we review current evidence implicating two groups of miRNAs - the miR-17 92 cluster and miR-200s - in the pathogenesis of PKD. We present a new hypothesis for cyst growth involving miRNAs and regulation of PKD gene dosage. We propose that manipulating miRNA function in an attempt to normalize PKD gene dosage represents a novel therapeutic strategy in PKD.
\end{abstract}

\section{Introduction}

PKD is characterized by the presence of innumerable, progressively-enlarging fluid-filled cysts in the renal parenchyma [1]. The cysts are derived from renal tubules and are lined by abnormally functioning and highly proliferative epithelial cells. The cysts increase in size overtime, compress surrounding normal nephrons, and cause inflammation, interstitial fibrosis and renal failure. Unfortunately, no FDA-approved therapy exists for prevention or retardation of cyst growth.

New evidence links miRNAs to the pathogenesis of kidney cyst growth. First, inhibition of miRNA biogenesis in maturing renal tubules produces numerous tubular and glomerular cysts [2]. Second, aberrant miRNA expression directly contributes to cyst growth in mouse models of PKD [3], and finally, miRNAs regulate the expression of many cystic kidney disease genes [2-4].

\section{Polycystic Kidney Disease (PKD)}

A. Genetics, clinical features and pathogenesis-PKD can be inherited in an autosomal dominant (ADPKD) or autosomal recessive (ARPKD) manner. The incidence of ADPKD is 1:400 -1:1000 live births. It is estimated that $\sim 600,000$ Americans and $\sim 12.5$

\footnotetext{
(C) 2013 Elsevier Ltd. All rights reserved.

Address correspondence to: Vishal Patel, M.D. Dept. of Internal Medicine/Nephrology 5323 Harry Hines Blvd, F5206 Dallas, TX, USA, 75390 Tel: 2146482754 Fax: 214-648-2071 Vishald.patel@utsouthwestern.edu.

Publisher's Disclaimer: This is a PDF file of an unedited manuscript that has been accepted for publication. As a service to our customers we are providing this early version of the manuscript. The manuscript will undergo copyediting, typesetting, and review of the resulting proof before it is published in its final citable form. Please note that during the production process errors may be discovered which could affect the content, and all legal disclaimers that apply to the journal pertain.

Conflicts of Interest: None
} 
million people world-wide are affected by ADPKD. Nearly $50 \%$ of individuals with ADPKD will eventually develop end-stage renal disease (ESRD). ADPKD is the 4th largest and most common genetic cause of ESRD in the USA. It accounts for $\sim 5 \%$ of people on dialysis or requiring kidney transplantation. ADPKD primary manifests in adults, and affects all races and both sexes equally. The clinical presentation of ADPKD can range from asymptomatic individuals who are diagnosed on accidental ultrasound screening to patients who present with ESRD and large abdominal masses due to enlarged kidneys. The clinical features of ADPKD include innumerable bilateral kidney cysts causing massive kidney enlargement ( $100 \%$ of patients), cysts in the bile ducts ( $80 \%$ of patients), pancreas, seminal vesicles, choroid plexus, and thyroid ( $10 \%$ of patients). Additional features include hypertension ( $\sim 80 \%$ of patients), mitral valve prolapse, mitral and aortic regurgitation ( $25 \%$ of patients), intracranial aneurysms ( $10 \%$ of patients), inguinal hernia, colic diverticulosis and kidney stones ( $\sim 10 \%$ of patients) [5].

ADPKD is caused by mutations of two genes: majority of patients ( $\sim 85 \%)$ carry mutations of $P K D 1$, which encodes a protein called polycystin-1. The remaining patients $(\sim 15 \%)$ carry mutations of $P K D 2$, which encodes a protein called polycystin-2. Every cell in an individual affected with ADPKD carries an inherited (germline) mutation of the PKD gene. Despite this, cysts form only in very few numbers of nephrons. In addition to the germline mutation, it is believed that ADPKD patients develop a somatic mutation of the second normal allele later in life, referred to as the 'second hit'. The 'second hit' results in the loss of both alleles, which then initiates cyst formation. Animal studies have shown non-genetic factors such as acute kidney injury also modulates the phenotype severity in PKD [6].

ARPKD is a rare condition with an incidence of $\sim 1: 20,000$ live births. ARPKD is caused by homozygous mutations in $P K H D 1$, which encodes fibrocystin (also known as polyductin). About one third of patients die immediately after birth due to respiratory difficulties from enlarged kidneys. Those that survive develop large liver cysts due to expansion of cells lining the bile ducts. These patients later develop liver fibrosis and portal hypertension. More than $50 \%$ of ARPKD patients develop ESRD before the age of 20 years.

PKD belongs to a new class of diseases called 'ciliopathies' [7]. The primary cilium is a hair-like structure present on the surface of most cells in the body. In the kidney, primary cilium is present on the apical surface of most renal tubule epithelial cells. Renal cilia project into the tubular lumen and are believed to sense urine flow. Fluid flows over the apical surface of the cells, bends the primary cilium, and produces an increase in intracellular calcium concentration, which regulates multiple cell signaling pathways. Three lines of evidence link primary cilia to cyst pathogenesis: First, polycystin-1, polycystin-2, fibrocystin and other proteins mutated in cystic kidney diseases are located in primary cilium. Second, $P k d 1$ and $P k d 2$ mutant cells contain dysfunctional primary cilia evidenced by a failure to increase calcium concentration in response to fluid flow [8]. Third, kidney tubule-specific loss of primary cilia produces PKD in mice [6,9].

B. Prognosis of PKD-Most conditions that cause ESRD such as glomerulonephritis, diabetes and hypertension affect the glomerular structure. Therefore, estimating glomerular filtration rate (GFR) - with tests such as serum creatinine measurements - is a reasonable 
way to monitor the progression of these diseases. In contrast, ADPKD affects the renal tubules whereas the glomerulus is structurally intact. Therefore, using GFR to monitor the progression of ADPKD may be problematic. To document the natural course of ADPKD and to identify reliable markers of disease progression, a multi-center prospective study called 'Consortium for Radiologic Imaging Studies of Polycystic Kidney Disease (CRISP)' was initiated in 2000. This study formally, but not surprisingly, showed that baseline kidney volume and rate of increase in kidney volume are better indicators of disease progression than baseline GFR in ADPKD [10]. In recent years, the scope of the CRISP study has been expanded. The investigators are now performing genotype-phenotype correlations, attempting to identify novel biomarkers of disease progression in urine and blood samples of patients, and using advanced radiological techniques to quantify the influence of renal blood flow, number of cysts, kidney volume and topography on the clinical course of ADPKD.

\section{MicroRNAs and PKD}

A. miRNAs are essential for renal tubule development-MicroRNAs (miRNAs) are small non-coding RNAs that constitute the endogenous RNA-interference pathway in plants and animals [11,12]. Over 1000 different miRNAs are encoded by the human genome, the majority of which are conserved in other species. miRNAs are initially transcribed as precursor transcripts called primary miRNAs (pri-miRs). Pri-miRs are then sequentially processed by enzymes Drosha and Dicer to form mature miRNAs. Nucleotides $2-8$ at the 5 ' end of a mature miRNA is referred to as the seed sequence. Base pairing between seed sequence and sequences primarily located in the 3'-UTRs of target mRNAs disrupts the translation of target mRNAs. In this manner, miRNAs function as sequencespecific inhibitors of gene expression.

miRNAs have emerged as critical regulators of development of various organs, including the kidney. Kidney development involves a series of reciprocal interactions between the ureteric bud (UB) and the metanephric mesenchyme (MM). Signals arising from the UB induce the $\mathrm{MM}$ to from renal vesicles (RV), which eventually become the nephrons and glomeruli. Conversely, signals arising from the MM induce UB branching, which eventually become collecting ducts. Through this repetitive process the kidney is made up of $\sim 1$ million nephrons. miRNAs play essential roles in all stages of kidney development [13]. Inactivation of Dicer from nephron progenitor cells leads to premature termination of RV formation causing renal agenesis [14]. Inactivation of Dicer from UBs leads to premature termination of UB branching also causing renal agenesis [15]. Studies from our group have shown that inactivation of Dicer at later stages of renal tubule development - subsequent to $\mathrm{RV}$ formation and UB branching - produces kidney cysts [2]. The cysts arise in a cell autonomous manner directly linking miRNAs to renal tubule maturation. Conversely, dysregulated miRNA expression is observed in established animal models of PKD in which the miRNA biogenesis pathway is genetically unperturbed [16,17]. Collectively, these studies suggest that miRNAs play a key role in the maintenance of renal tubule integrity, and aberrant miRNA expression contributes to cyst formation and cyst growth.

\section{B. miRNAs regulate the expression of cystic kidney disease genes-Two} groups of miRNAs - the miR-17 and related miRNAs, and the miR-200 miRNA family - 
have been shown to function as novel regulators of PKD gene dosage $[2,4]$. These observations raise an intriguing possibility that miRNAs not only regulate renal tubule development but also contribute to cyst pathogenesis through regulation of PKD genes.

The miR-17 and related miRNAs are derived from three miRNA clusters: miR-17 92 cluster, the miR-106a-363 cluster and the miR-106b 25 cluster. The genomic organization and coding sequences of these miRNA clusters are evolutionarily conserved in vertebrates. Based on their seed sequence, miRNAs derived from the three clusters can be classified into four families: the miR-17 family, miR-18 family, miR-19 family and miR-25 family. Since members of each family have an identical seed sequence, they are predicted to target the same mRNAs. Interestingly, even though each family is predicted to repress different sets of mRNAs, many putative targets function in the same cell signaling pathway. miR-17 92 and related clusters are enriched in developing tissues and are essential for heart and lung development [18]. Two major lines of evidence implicate miR-17 and related miRNAs in the pathogenesis of various cancers $[19,20]$. First, these miRNAs are amplified in numerous human cancers, promote proliferation [21] and cause tumour growth in vivo [19]. Second, the oncogenic transcription factor c-Myc binds to the promoter of miR-17 92 and induces its transcription [22]. The miRNAs, in turn, repress several tumor suppressors, thus, aiding in tumor growth. miR-17 and related miRNAs have also been implicated in the pathogenesis of PKD [3]. We have recently shown that miRNAs derived from the miR-17 92 cluster are up-regulated in multiple mouse models of PKD [3]. Similar to many forms of cancer, cMyc-mediated transcriptional activation may be the mechanism underlying up-regulation of miR-17 92 in PKD. To test whether miR-17 92 directly contributed to cyst formation or cyst growth, we generated transgenic mice with kidney-specific gain-of-function and lossof-function alleles of miR-17 92. Kidney-specific transgenic overexpression of miR-17 92 produced kidney cysts. Conversely, inactivation of miR-17 92 in renal tubules of kidneyspecific Kif3a mutant mice (Kif3a-mutant), an animal model of PKD, ameliorated the cystic phenotype. These studies demonstrate that miR-17 92 is a novel regulator of kidney cyst growth, and provide genetic proof-of-principle for inhibition of miR-17 92 as a novel therapeutic approach for PKD. Our work suggests that the mechanism by which miR-17 92 promotes cyst growth may involve increased proliferation and inhibition of PKD genes (Figure 1) [3]. 3'-UTRs of the ADPKD genes $P K D 1$ and $P K D 2$ contains conserved binding sites for members of the miR-17 family. $H N F-1 \beta$, an epithelium-specific transcription factor, harbors a conserved binding site for miRNAs belonging to the miR-25 family. Mutations of $H N F-1 \beta$ produce a rare clinical syndrome called renal cysts and diabetes (RCAD) [23]. $H N F-1 \beta$ promotes the expression of $P K H D 1, P K D 2$ and numerous other cystic kidney disease genes [24]. In cultured cells, miR-17 represses $P k d l$ and $P k d 2$ while miR-25 family members repress Hnf-1 $\beta$ through interaction with binding sites in their 3'-UTRs. In mice, overexpression of miR-17 92 is associated with increased proliferation of renal epithelial cells and down-regulation of $P k d 1, P k d 2$ and $H n f-1 \beta$. Conversely, inactivation of miR-17 92 in renal tubules of Kif3a-mutant mice is associated with decreased proliferation of cyst epithelial cells and up-regulation of $P k d 1, P k d 2$ and Hnf-1 $\beta$. Additionally, miR-17 and related families are predicted to target several other genes that are mutated in humans with cystic kidney disease and developmental disorders of the kidney (Figure 2). Moreover, several genes that are essential for the normal function of primary cilium are also predicted 
targets of the miR-17 and related miRNA families. Thus, miR-17 92 may promote disease progression in PKD through repression of a network of cystic kidney disease genes.

The miR-200 family consists of five miRNAs - miR-200a, miR-200b, miR-200c, miR-429 and miR-141 - that are derived from two clusters: the miR-200b 429 cluster and miR-200c 141 cluster. The miR-200s are enriched in epithelial organs such as kidney and lung [2]. A major function of miR-200 is to prevent epithelial-to-mesenchymal transition (EMT) [13]. We studied the function of miR-200s in renal tubules by generating kidneyspecific Dicer mutant mice. Inactivation of Dicer was associated with downregulation of all members of the miR-200 family, and formation of numerous kidney cysts. However, downregulation of miR-200 did not induce EMT in kidney cysts or cultured renal epithelial cells. miR-200s are also downregulated in cystic kidneys from mouse models of PKD, where EMT is not observed. By combining gene expression profiling with 3'-UTR analysis, we found that miR-200 regulates the expression of cystic kidney disease genes, thus identifying a novel function for the miR-200 family in thekidney [2]. Specifically, we found that $P K D 1$ harbors two conserved binding sites for miR-200 in its 3'-UTR, and miR-200b/c/429 repress the expression of $P k d l$ through interaction with the binding sites [2]. Additionally, miR-200 is predicted to target GLIS2, mutations of which cause a rare form of cystic kidney disease called nephronopthisis, and $H N F-1 \beta$. These results suggest that miR-200s maintain renal epithelial homeostasis and renal tubule integrity through an EMT-independent process involving the regulation of cystic kidney disease genes.

\section{C. miRNA-mediated regulation of PKD gene dosage: a new mechanism for}

cyst growth?-How would miRNA-mediated repression of genes that are already mutated in cystic kidney disease still contribute to disease progression? Accumulating evidence suggests that, rather than a complete loss-of-function, altered dosage of cystic kidney disease genes underlies disease progression in some forms of PKD. Molecular diagnostics surveys have shown that $\sim 26$ to $37 \%$ of ADPKD patients harbor non-truncating mutations of $P K D 1$ [25]. In contrast to the truncating mutations, which produce loss-of-function alleles, the non-truncating mutations are predicted to produce incompletely penetrant and hypomorphic alleles of $P K D 1$. Rossetti et al, have studied one such mutation - the R3277C mutation of $P K D 1$ - in greater detail [26]. They found that family members that were heterozygous for this mutation developed a mild form of PKD whereas family members that were homozygous for this mutation developed typical PKD. However, ADPKD patients that co-inherited the R3277C mutation with a truncating mutation of PKD1 developed earlyonset, very severe form of PKD [26]. Similar results were obtained in mice harboring the human-equivalent mutation of R3277C [27]. These results provide strong evidence in support of the 'gene dosage' hypothesis. The hypothesis states that a critical threshold of functional $P K D I$ dosage is needed to prevent cyst formation. Lowering PKDI expression below this threshold produces kidney cysts. Severity of the cystic phenotype depends upon the dosage of $P K D 1$.

Disease progression in ADPKD may also be influenced by the dosage of cystic kidney disease genes other than PKD1. ADPKD patients who co-inherit heterozygous mutations of both $P K D 1$ and $P K D 2$ have a severe cystic phenotype compared to family members with single gene mutations [28]. Similarly, trans-heterozygous $P k d 1$ and $P k d 2\left(P k d 1^{+/-} ; P k d 2^{+/-}\right)$ 
mutant mice display a more severe cystic phenotype compared to $P k d 1^{+/-}$or $P k d 2^{+/-}$mice [29]. Decreased expression of $H N F-1 \beta$ and its target genes is observed in ADPKD [30]. ADPKD patients who carry heterozygous mutations of both $P K D 1$ and $H N F-1 \beta$ develop a more severe kidney abnormalities compared to siblings with single gene mutations [31]. Lowering $P k d 1$ or $P k d 2$ expression in $P k h d 1^{-/-}$mice produces a more severe cystic phenotype [32-34], suggesting that decreased dosage of ADPKD genes may also influence disease progression in ARPKD. Thus, PKD1, PKD2, PKHD1, HNF- $1 \beta$ and $H N F-1 \beta$ target genes genetically interact and collectively influence the cystic phenotype. Environmental factors and genetic factors that decrease the dosage of these genes may act as modifiers of disease progression in PKD. Paradoxically, increased $P K D 1$ gene dosage also produces kidney cysts in mice [35], suggesting that $P K D 1$ expression must be tightly regulated for normal renal tubule development and maintenance of tubular integrity.

It is tempting to speculate that miRNAs are genetic factors that modify disease progression in PKD through regulation of a network of cystic kidney disease genes. For example, upregulation of miR-17 and related miRNAs in cystic kidneys could repress multiple cystic kidney disease genes and thereby, aggravate the cystic phenotype. Conversely, downregulation of miR-200 in cystic kidneys may contribute to cyst growth via upregulation of PKDl and/or other cystic kidney disease genes.

\section{Potential of miRNAs as therapeutic targets in PKD-Management of PKD} patients consists of supportive measures such as analgesics for control of pain, antibiotics for cyst infection, and blood pressure control. Major advancements have been made towards identifying a cure for PKD. Based on their efficacy in animal models, two classes of drugs mTOR inhibitors and V2 receptor antagonists -have been tested in large human clinical trials. Rapamycin, an mTOR inhibitor, retards cyst growth in several animal models of PKD. Based on these promising results, clinical trials were performed to assess the efficacy of the mTOR inhibitors everolimus and sirolimus in humans with ADPKD [36,37]. Unfortunately, both clinical trials failed to show that mTOR inhibition retards cyst growth in humans.

Criticisms of these studies are that the sirolimus dose used was too low, the time of treatment was too short, and the enrolled patients had advanced PKD. cAMP levels are increased in cystic kidneys, and administration of Tolvaptan, a vasopressin $\mathrm{V} 2$ receptor antagonist, to animal models of PKD decreases cAMP levels and retards cyst growth [38]. Impressively, in a randomized clinical trial, tolvaptan retarded cyst growth in ADPKD patients [39]. A limitation of tolvaptan is that it has considerable side effects such as polyuria and increased thirst. Thus, adherence to a lifelong therapy may be difficult to achieve with tolvaptan, as evidenced by high patient dropout rates in the clinical trial [39]. Several clinical trials testing the efficacy of other classes of drugs are on-going, raising the hope for cure of PKD in the future.

Current therapeutic approaches target dysregulated cell signaling pathways downstream of the PKD genes [1]. An alternate therapeutic strategy could be to improve the expression of PKD genes and target downstream pathways at the same time. In this regard, miR-17 and related miRNA families represent attractive therapeutic targets. These miRNAs not only repress the PKD genes, but also regulate cyst-promoting pathways, such as the mTOR pathway. Activity of miRNAs can be inhibited using oligonucleotides called antimirs or 
antagomirs [13]. Antimirs have a sequence that is complementary to a miRNA of interest. Base-pairing between the antimir and the targeted miRNA sequesters the miRNA and inhibits its activity. Antimirs are chemically-modified to enhance their stability, promote cellular uptake and increase the specificity and duration of miRNA inhibition. Antimirs against several miRNAs are in various stages of preclinical and clinical testing as therapeutic agents for a wide-range of diseases such as cancer, tissue fibrosis and Hepatitis $\mathrm{C}$ infection. A unique feature of antimirs is that they primarily accumulate in normal liver and kidney [40]. Therefore, a miRNA-based therapeutics approach holds great promise for liver and kidney diseases. In addition to identifying miRNAs that represent therapeutic targets, a significant challenge in PKD will be to pharmaceutically inhibit miRNAs in cystic kidneys. Unlike the normal kidney, several factors may impede delivery of antimirs to cystic kidneys. The enlarging cysts distort the surrounding blood vessels, which could reduce the delivery of antimirs to cyst epithelial cells. The majority of antimirs that are filtered by the kidney are reabsorbed by proximal tubules. Therefore, enough concentration of antimirs may not be delivered to cysts that arise in distal segments of the nephron. Careful in vivo studies will be needed to determine whether antimirs are delivered to kidney cysts.

\section{Conclusion}

PKD is a common genetic disorder for which no FDA-approved treatment currently exists. miRNAs have emerged as potential new regulators of disease progression in PKD. Specifically, two groups of miRNAs - the miR-17 and related miRNAs, and the miR-200 miRNA family - may modulate cyst growth through inhibition of a network of cystic kidney disease genes. Manipulating miRNA function in an attempt to normalize the expression of cystic kidney disease genes represents an exciting new therapeutic approach in PKD.

\section{Acknowledgments}

The work in our laboratory is supported by grants from the National Institutes of Health/National Institute of Diabetes and Digestive and Kidney Diseases (KO8 DK084311-01 to VP) and the UT Southwestern O'Brien Kidney Research Core Center (P30 DK079328). LN is supported by a T32 training grant (DK007257) from NIDDK.

\section{References}

1. Patel V, et al. Advances in the pathogenesis and treatment of polycystic kidney disease. Curr Opin Nephrol Hypertens. 2009; 18(2):99-106. [PubMed: 19430332]

2. Patel V, et al. MicroRNAs Regulate Renal Tubule Maturation through Modulation of Pkd1. J Am Soc Nephrol. 2012

3. Patel V, et al. miR-17 92 miRNA cluster promotes kidney cyst growth in polycystic kidney disease. Proc Natl Acad Sci U S A. 2013

4. Tran $\mathrm{U}$, et al. The RNA-binding protein bicaudal $\mathrm{C}$ regulates polycystin 2 in the kidney by antagonizing miR-17 activity. Development. 2010; 137(7):1107-1116. [PubMed: 20215348]

5. Grantham JJ. Clinical practice. Autosomal dominant polycystic kidney disease. N Engl J Med. 2008; 359(14):1477-1485. [PubMed: 18832246]

6 . Patel V, et al. Acute kidney injury and aberrant planar cell polarity induce cyst formation in mice lacking renal cilia. Hum Mol Genet. 2008; 17(11):1578-1590. [PubMed: 18263895]

7. Hildebrandt F, et al. Ciliopathies. N Engl J Med. 2011; 364(16):1533-1543. [PubMed: 21506742]

8. Nauli SM, Zhou J. Polycystins and mechanosensation in renal and nodal cilia. Bioessays. 2004; 26(8):844-856. [PubMed: 15273987] 
9. Lin F, et al. Kidney-specific inactivation of the KIF3A subunit of kinesin-II inhibits renal ciliogesis and produces polycystic kidney disease. Proc Natl Acad Sci U S A. 2003; 100(9):5286-5291. [PubMed: 12672950]

10. Grantham JJ, et al. Volume progression in polycystic kidney disease. N Engl J Med. 2006; 354(20):2122-2130. [PubMed: 16707749]

11. Bartel DP. MicroRNAs: genomics, biogenesis, mechanism, and function. Cell. 2004; 116(2):281297. [PubMed: 14744438]

12. Bartel DP. MicroRNAs: target recognition and regulatory functions. Cell. 2009; 136(2):215-233. [PubMed: 19167326]

13. Patel V, Noureddine L. MicroRNAs and fibrosis. Curr Opin Nephrol Hypertens. 2012; 21(4):410416. [PubMed: 22622653]

14. Ho J, et al. The pro-apoptotic protein Bim is a microRNA target in kidney progenitors. J Am Soc Nephrol. 2011; 22(6):1053-1063. [PubMed: 21546576]

15. Nagalakshmi VK, et al. Dicer regulates the development of nephrogenic and ureteric compartments in the mammalian kidney. Kidney Int. 2011; 79(3):317-330. [PubMed: 20944551]

16. Pandey $P$, et al. Microarray-based approach identifies microRNAs and their target functional patterns in polycystic kidney disease. BMC Genomics. 2008; 9:624. [PubMed: 19102782]

17. Pandey $P$, et al. Systems biology approach to identify transcriptome reprogramming and candidate microRNA targets during the progression of polycystic kidney disease. BMC Syst Biol. 2011; 5:56. [PubMed: 21518438]

18. Ventura A, et al. Targeted deletion reveals essential and overlapping functions of the miR-17 through 92 family of miRNA clusters. Cell. 2008; 132(5):875-886. [PubMed: 18329372]

19. Conkrite K, et al. miR-17 92 cooperates with RB pathway mutations to promote retinoblastoma. Genes Dev. 2011; 25(16):1734-1745. [PubMed: 21816922]

20. Mendell JT. miRiad roles for the miR-17-92 cluster in development and disease. Cell. 2008; 133(2):217-222. [PubMed: 18423194]

21. Cloonan N, et al. The miR-17-5p microRNA is a key regulator of the G1/S phase cell cycle transition. Genome Biol. 2008; 9(8):R127. [PubMed: 18700987]

22. O'Donnell KA, et al. c-Myc-regulated microRNAs modulate E2F1 expression. Nature. 2005; 435(7043):839-843. [PubMed: 15944709]

23. Igarashi $\mathrm{P}$, et al. Roles of HNF-1beta in kidney development and congenital cystic diseases. Kidney Int. 2005; 68(5):1944-1947. [PubMed: 16221171]

24. Gresh L, et al. A transcriptional network in polycystic kidney disease. EMBO J. 2004; 23(7):16571668. [PubMed: 15029248]

25. Rossetti S, et al. Comprehensive molecular diagnostics in autosomal dominant polycystic kidney disease. J Am Soc Nephrol. 2007; 18(7):2143-2160. [PubMed: 17582161]

26. Rossetti $\mathrm{S}$, et al. Incompletely penetrant PKD1 alleles suggest a role for gene dosage in cyst initiation in polycystic kidney disease. Kidney Int. 2009; 75(8):848-855. [PubMed: 19165178]

27. Hopp K, et al. Functional polycystin-1 dosage governs autosomal dominant polycystic kidney disease severity. J Clin Invest. 2012; 122(11):4257-4273. [PubMed: 23064367]

28. Pei Y, et al. Bilineal disease and trans-heterozygotes in autosomal dominant polycystic kidney disease. Am J Hum Genet. 2001; 68(2):355-363. [PubMed: 11156533]

29. Wu G, et al. Trans-heterozygous Pkd1 and Pkd2 mutations modify expression of polycystic kidney disease. Hum Mol Genet. 2002; 11(16):1845-1854. [PubMed: 12140187]

30. Song X, et al. Systems biology of autosomal dominant polycystic kidney disease (ADPKD): computational identification of gene expression pathways and integrated regulatory networks. Hum Mol Genet. 2009; 18(13):2328-2343. [PubMed: 19346236]

31. Bergmann C, et al. Mutations in multiple PKD genes may explain early and severe polycystic kidney disease. J Am Soc Nephrol. 2011; 22(11):2047-2056. [PubMed: 22034641]

32. Fedeles SV, et al. A genetic interaction network of five genes for human polycystic kidney and liver diseases defines polycystin-1 as the central determinant of cyst formation. Nat Genet. 2011; 43(7):639-647. [PubMed: 21685914] 
33. Garcia-Gonzalez MA, et al. Genetic interaction studies link autosomal dominant and recessive polycystic kidney disease in a common pathway. Hum Mol Genet. 2007; 16(16):1940-1950. [PubMed: 17575307]

34. Kim I, et al. Fibrocystin/polyductin modulates renal tubular formation by regulating polycystin-2 expression and function. J Am Soc Nephrol. 2008; 19(3):455-468. [PubMed: 18235088]

35. Pritchard L, et al. A human PKD1 transgene generates functional polycystin-1 in mice and is associated with a cystic phenotype. Hum Mol Genet. 2000; 9(18):2617-2627. [PubMed: 11063721]

36. Walz G, et al. Everolimus in patients with autosomal dominant polycystic kidney disease. N Engl J Med. 2010; 363(9):830-840. [PubMed: 20581392]

37. Serra AL, et al. Sirolimus and kidney growth in autosomal dominant polycystic kidney disease. N Engl J Med. 2010; 363(9):820-829. [PubMed: 20581391]

38. Gattone VH 2nd, et al. Inhibition of renal cystic disease development and progression by a vasopressin V2 receptor antagonist. Nat Med. 2003; 9(10):1323-1326. [PubMed: 14502283]

39. Torres VE, et al. Tolvaptan in patients with autosomal dominant polycystic kidney disease. N Engl J Med. 2012; 367(25):2407-2418. [PubMed: 23121377]

40. Obad S, et al. Silencing of microRNA families by seed-targeting tiny LNAs. Nat Genet. 2011; 43(4):371-378. [PubMed: 21423181] 


\section{Highlights}

- PKD, one of the most common human genetic disorders, causes numerous kidney cysts and renal failure

- Currently, no definitive therapy exists to retard cyst growth

- miR-17 92, an oncogenic miRNA cluster, is a novel regulator of kidney cyst growth

- miRNAs may modulate cyst growth through repression of a network of cystic kidney disease genes

- Attempting to normalize the expression of cystic kidney disease genes by modulating miRNA function represents a novel therapeutic strategy 


\section{Normal kidney}

\section{miR-17 92}

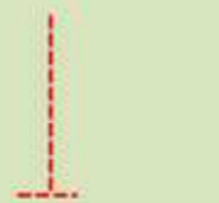

PKD genes

\section{Prevention of cyst formation}

\section{PKD}

\section{miR-17 92}

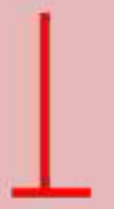

PKD genes

\section{Cyst formation}

Figure 1. The proposed mechanism by which miR-17 92 promotes cyst growth in PKD miR-17 92 promotes proliferation and cyst growth potentially through post-transcriptional inhibition of PKD genes and other targets. In wild-type adult kidneys, this mechanism would be restrained by the normally low levels of miR-17 92. However, in cystic kidneys, increased levels of miR-17 92 will down-regulate its targets, which in turn promotes proliferation and cyst growth. 


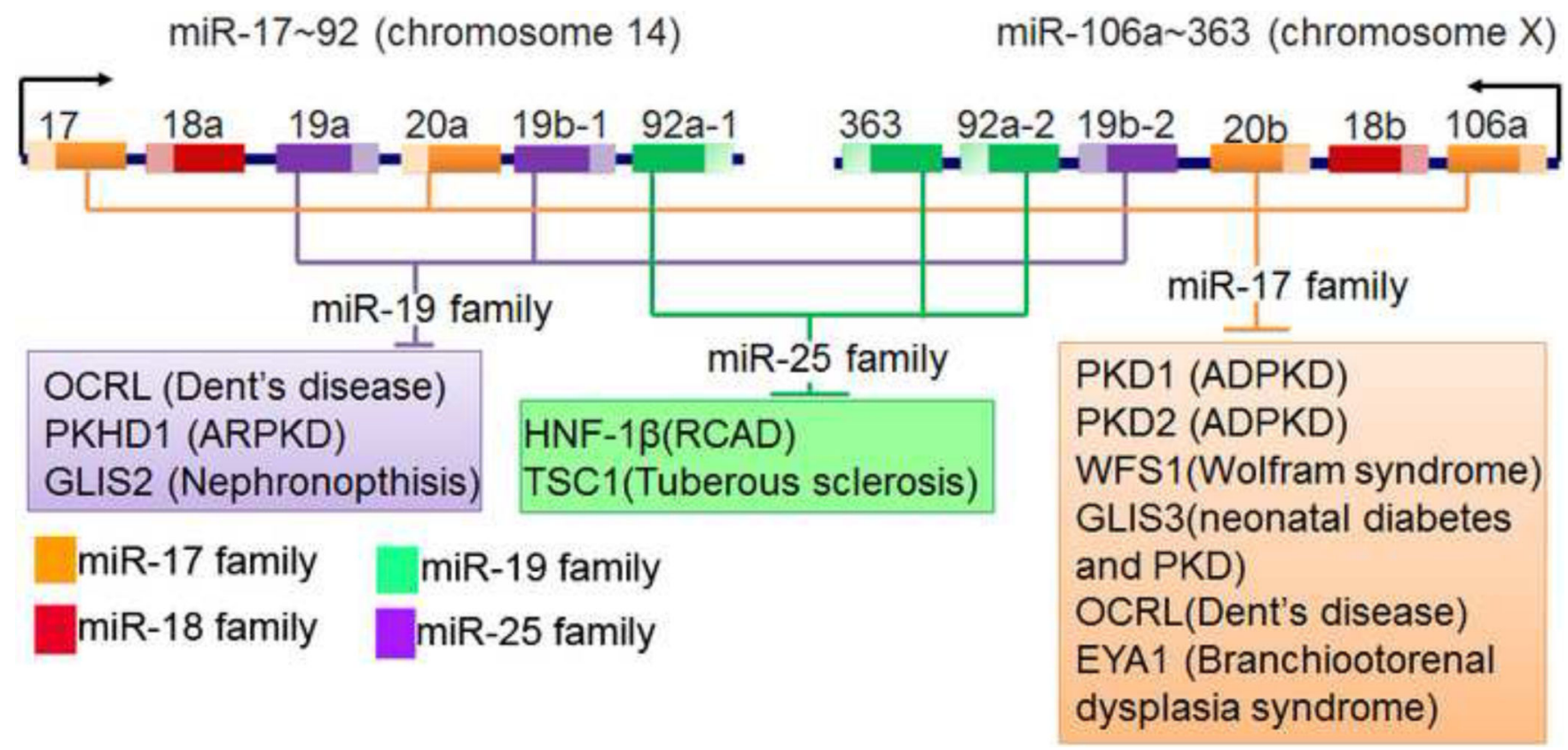

Figure 2.

miR-17 and related miRNA families are predicted to target genes that are mutated in cystic kidney diseases and developmental disorders of kidney. 


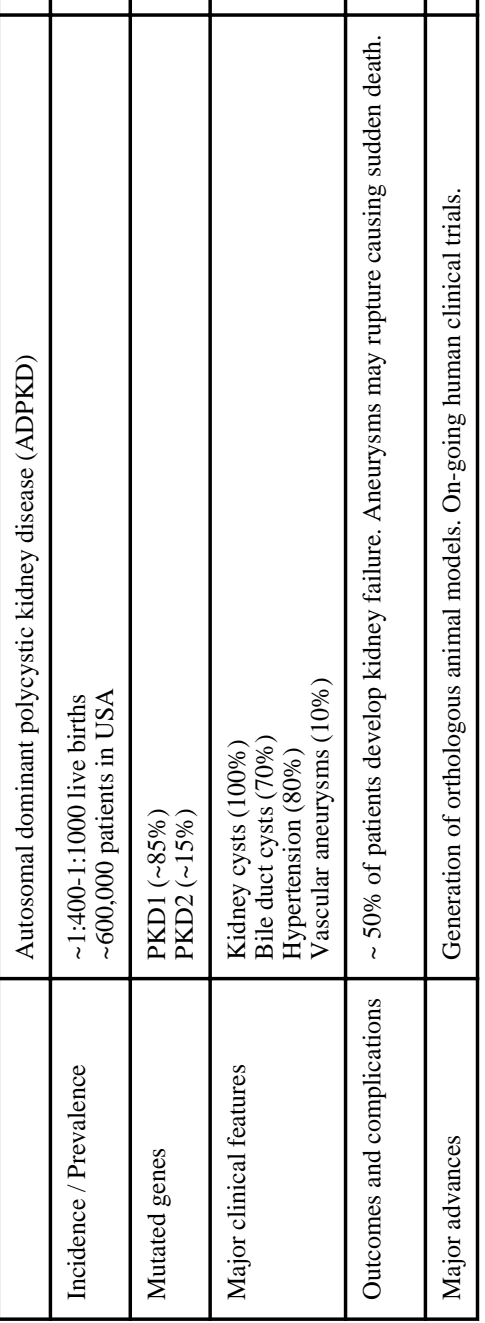

